\title{
DESIGN AND REALIZATION OF DELAY MAPPING RECEIVER BASED ON GPS FOR SEA SURFACE WIND MEASUREMENT
}

\author{
Hu Ronglei Yang Dongkai Zhang Qishan Zhang Yiqiang \\ Beihang University, Xueyuan Road, Haidian District, Beijing, P.R.China, 100083
}

\begin{abstract}
The Delay Mapping Receiver (DMR) is used for receiving and processing the reflected GPS signal to get the information of sea surface wind by recording and matching the data with the theoretical model. The hardware architecture and software design are described in detail in this paper. The test results at near sea of Tianjin of China are provided, which prove that the design of DMR is successful and the collected data are useful for the sea surface wind measurement.
\end{abstract}

\section{KEY WORDS}

Remote Sensing, Delay Mapping Receiver, Global Positioning System, Sea Wind Measurement

\section{INTRODUCTION}

Global Positioning System (GPS) has been successfully used in studying humidity and temperature of atmosphere besides positioning and navigation. But only the direct GPS signal is analyzed for all of these applications. In general, the reflected GPS signal is not used for positioning and sometimes it worsen the positioning precision. However, it has been discovered that the reflected GPS signal from the sea surface contains information about sea surface roughness, which is related to the wind speed and wind direction of sea surface. The cross-correlation power curve of the reflected GPS signals will have a different shape for different sea surface roughness. The principle is shown in Figure 1. In ideal surface state (specular reflection), the shape of the cross-correlation power curve is very sharp triangle. It becomes wider and smoother as the roughness of the reflecting surface increases. The sea surface wind speed and direction can be determined by matching the recorded shape of this cross-correlation power curve to that predicted by theoretical models as a function of wind speed and direction. 


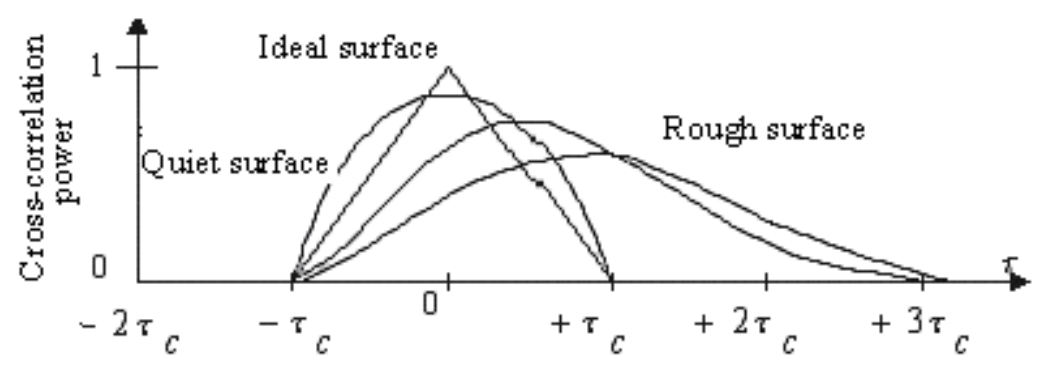

Figure 1 Cross-correlation power curve of reflected GPS signal

In comparison to conventional scatterometers, the new method using reflected GPS signal has several advantages: (1) This method needs GPS satellites as transmitter, which will obviously reduce the cost, complexity, size, and power requirement. (2) Spread spectrum techniques applied in GPS signal makes the receiver easily acquire and track reflected signal with lower SNR in airborne DMR. (3) Besides the GPS signal, GALILEO signal can also be used in the similar DMR device, i.e. there are more than one kind of signal source.

Delay Mapping Receiver (DMR) is developed to implement the GPS reflected signal collection and processing. The hardware, software design are discussed individually. And the test results are given in the final.

\section{HARDWARE DESIGN OF DMR}

The GPS satellites signal is right-hand circularly polarized (RHCP), but the reflected signal is left-hand circularly polarized (LHCP). The two kinds of signal can't be received using one antenna because RHCP antenna restrains LHCP signal, and vice versa. The commercial double-antenna GPS receiver process the same kind of signal with different parameters, it can't distinguish direct signal and reflect signal from each other. In this design two omnidirectional antennas and two RF front ends are used for DMR. One RHCP antenna is equipped to point to sky with one front end receiving and processing direct GPS signal, one LHCP antenna point to sea surface with another front end receiving and processing reflected GPS signal. The direct signal is used for positioning, and providing essential data for calculating code chip delay of reflected signal from sea surface. The hardware architecture of DMR based on commercial GPS receiver chipset is shown in Figure 2.

\section{Antenna module}

RHCP and LHCP antenna are included in the antenna modules. The signal reflected from sea surface is weaker than direct GPS signal, so we must take measures to improve the SNR of 
reflected signal. In the design we augment the LHCP antenna gain, select low-noise-coefficient RF amplifier and prolong accumulated data time. The flight tests we have done prove that the highest SNR of reflected signal can reach $22 \mathrm{~dB}$ when the flight altitude is about 1000 meters. The LHCP antenna can restrain RHCP signal at least $15 \mathrm{~dB}$.

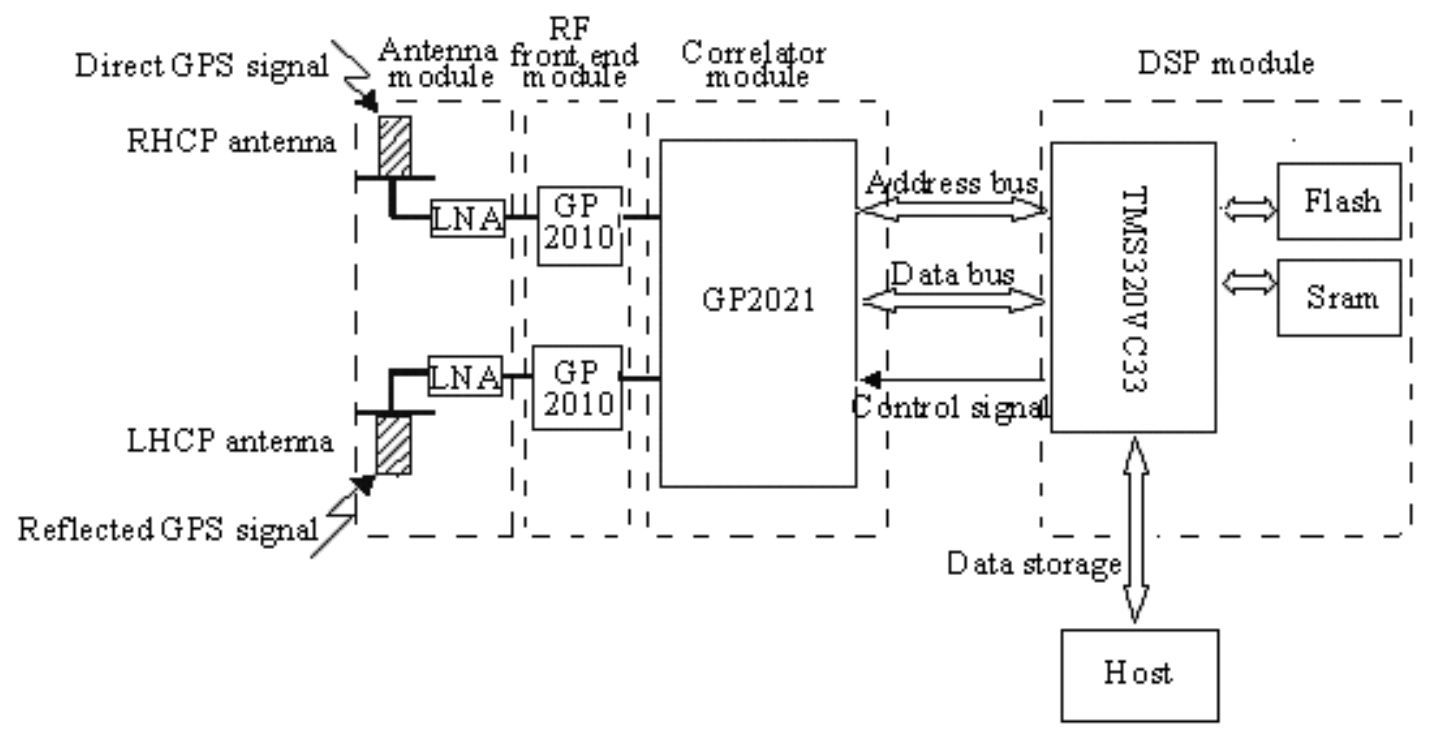

Figure 2. The hardware architecture of DMR

\section{RF front end module}

Two RF front ends are used in this DMR. One is used to receive signal from RHCP antenna and another is used to receive signal from LHCP antenna. The commercial GP2010 chip from Zarlink is selected as RF front end, shown in Figure 3. A 10MHz Temperature Compensated Crystal Oscillator (TCXO) is used as reference oscillator, which has high frequency stability. GPS signal carrier frequency down conversion from $1575.42 \mathrm{MHz}$ to $4.039 \mathrm{MHz}$ is through three stages. In the first stage the on-chip PLL generates the first local-oscillator frequency at $1400 \mathrm{MHz}$. The output of mixer is $175.42 \mathrm{MHz}$. In the second stage $140 \mathrm{MHz}$ frequency is generated and the output is $35.42 \mathrm{MHz}$, which is passed through an external SAW filter. The output of the filter then feeds the main IF amplifier with two Auto Gain Control (AGC) units. The third mixer with local oscillator at $31.111 \mathrm{MHz}$ gives a final IF at $4.309 \mathrm{MHz}$. There is an on-chip filter that provides filtering centered on $4.309 \mathrm{MHz}$ following the third mixer. A/D controller is used to produce a 2-bit digital output from the final IF signal.

The EMC problem must be solved in the proposed DMR design because there are two RF front ends, which influenced each other. We take such measures as isolating analog ground and digital ground, adding RF shield. The SNR of DMR can be improved $2 \mathrm{~dB}$ after all these methods are applied. 


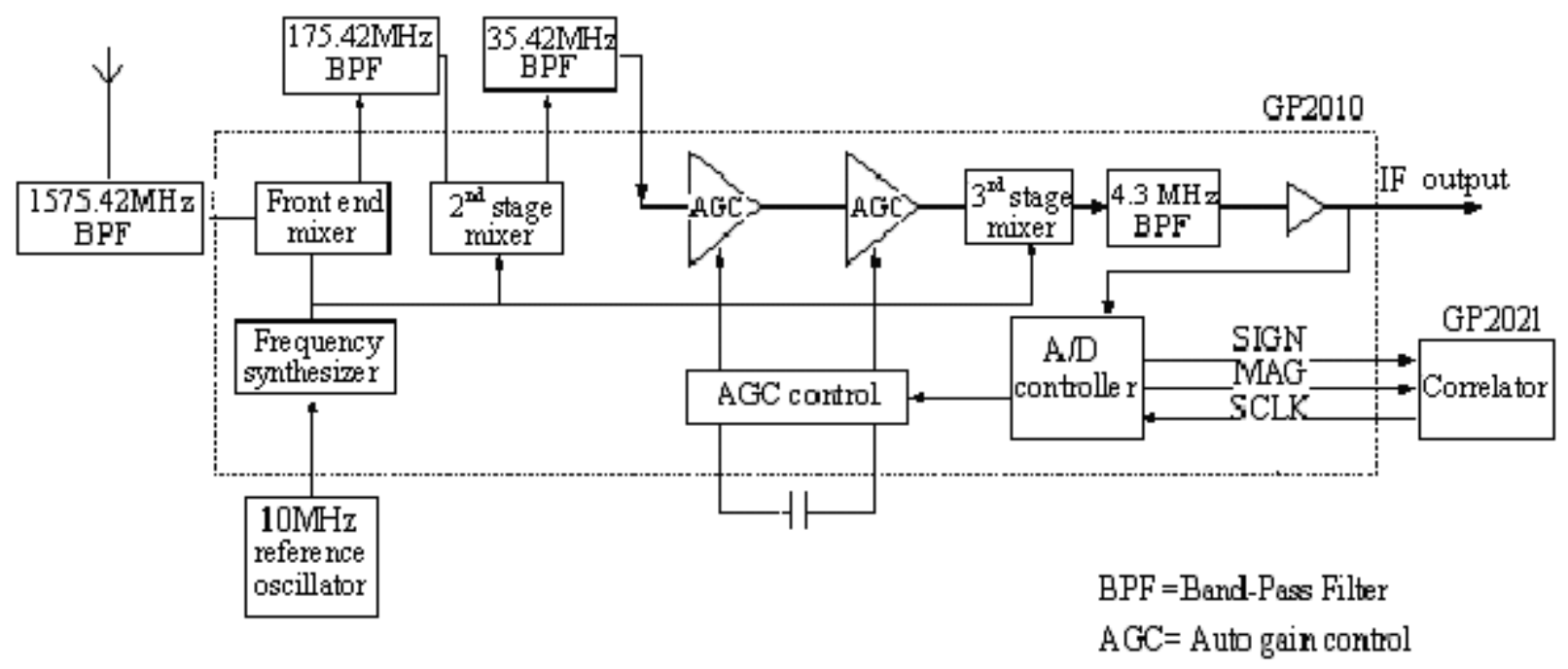

Figure 3. Block diagram of front end

\section{Correlator module}

Commercial GP2021 chip from Zarlink is selected as correlator. It incorporates 12 correlation channels for GPS C/A code base band digital signal together with dual UART, real time clock, etc. GP2021 generates a replica of satellites code to be used for computing of correlation power with received data. The correlation results in the GP2021 form the accumulated data and are transferred to DSP module to control the software signal tracking loops.

When GP2010 is used in conjunction with GP2021, one of GP2010 send a 40MHz clock to GP2021 by pin CLK_I and CLK_T, then the GP2021 provides a sampling clock SAMPCLK, i.e. $5.714 \mathrm{MHz}$. This converts the IF signal to 2-bit digital output at $1.405 \mathrm{MHz}$ and TTL levels. Figure 4 shows the connections between the two GP2010 and GP2021. There are two sets of signal inputs, MAG0, SIGN0 and MAG1, SIGN1 on GP2021. In this design MAG0 and SIGN0 are for RHCP antenna signal, MAG1 and SIGN1 are for LHCP antenna signal. Each GP2021 channel can be selected to process satellite signal from RHCP or LHCP antenna by configuring its register.

\section{DSP module}

DSP module includes DSP chip and its peripheral chips. TMS320VC33 from Texas Instruments is selected as processor for floating point operation. The processor read the accumulated data from GP2021 through address bus and parallel data bus to capture and track GPS satellites by controlling the code loop and carrier loop. RAM in the design includes embedded 34KB in processor and SDRM IS631v1024 as extended for temporary data. Flash AM29LV040 is used for processor boot and application program. The data processed by DMR 
will be sent to host via the UART of GP2021.

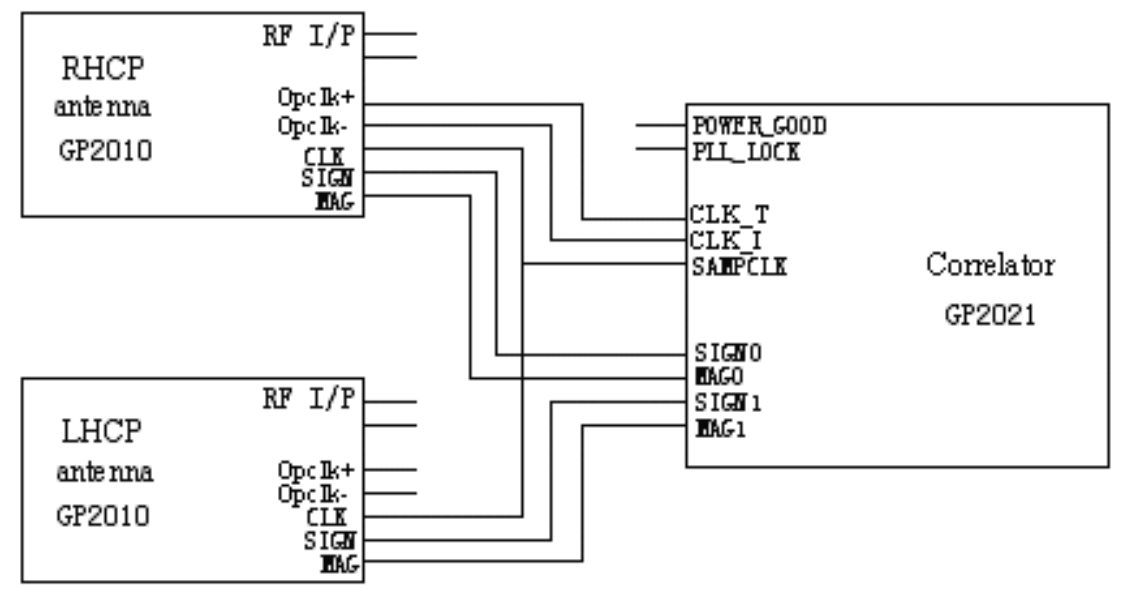

Figure 4. Connection between GP2010 and GP2021

\section{SOFTWARE DESIGN OF DMR}

\section{Software module}

DMR system software includes the following five parts: GPS signal processing, hardware control, GPS navigation message decoding, floating point navigation calculations and communications interface.

We define the correlator channels processing RHCP antenna signal as direct signal channel and that processing LHCP antenna signal as reflected signal channel. GPS signal processing module is for reading I and Q value (In-phase and quadrature sample are accumulated over $1 \mathrm{~ms}$ ) from direct and reflected signal channels, and judging the capturing and tracking procedure. Hardware control module is used for adjusting the value of code digital control oscillator (DCO) and carrier DCO according to the output of software discriminator. At the same time, it is used to configure reflected signal channels and adjust parameters according to Doppler frequency shift and carrier phase of direct signal channels. GPS navigation message decoding module is used for getting the essential parameters according to the format of the navigation data. Floating point navigation calculations module is used for calculating pseudorange for every satellite and then calculating the user position. Communications interface module is used for sending information to host according to certain format, including navigation and positioning results, correlation power, delay code chip, etc.

\section{Software operation mode}


Two operation modes are defined for DMR: parallel mode and serial mode. Parallel DMR (PDMR) only tracks 1 or 2 satellites via direct signal. Therefore it can't provide user with positioning calculations. Serial DMR (SDMR) provides user with positioning information and correlation power of reflected signal at the same time. Its working flow is shown as follows. (1) DSP configures correlator Channel 1-6 as direct signal channel for processing direct GPS signal, channels 7-12 as reflected signal channels for processing reflected signal from sea surface. (2) DSP read accumulated data from direct signal channels of GP2021 and process these data to perform code loop and carrier loop tracking, bit and frame synchronizing, navigation message decoding and positioning. DSP calculates also C/A code chip delay of reflected signals of channel 1-6 respectively. (3) DSP configures channel 7-12 of correlator using the satellites parameters including code phase and carrier frequency from channel 1-6, and adjusts these parameters according to Doppler frequency shift and carrier phase of channel 1-6. (4) DSP configures channel 7-12 using each delay half code chip numbered 0,1,2...32 which begin at the specular reflection delay code chip and step in half code chip, and then reads cross-correlation power for $1 \mathrm{~ms}$ at each step. The correlation power is sent to host once per second. The SDMR is shown in Figure 5.

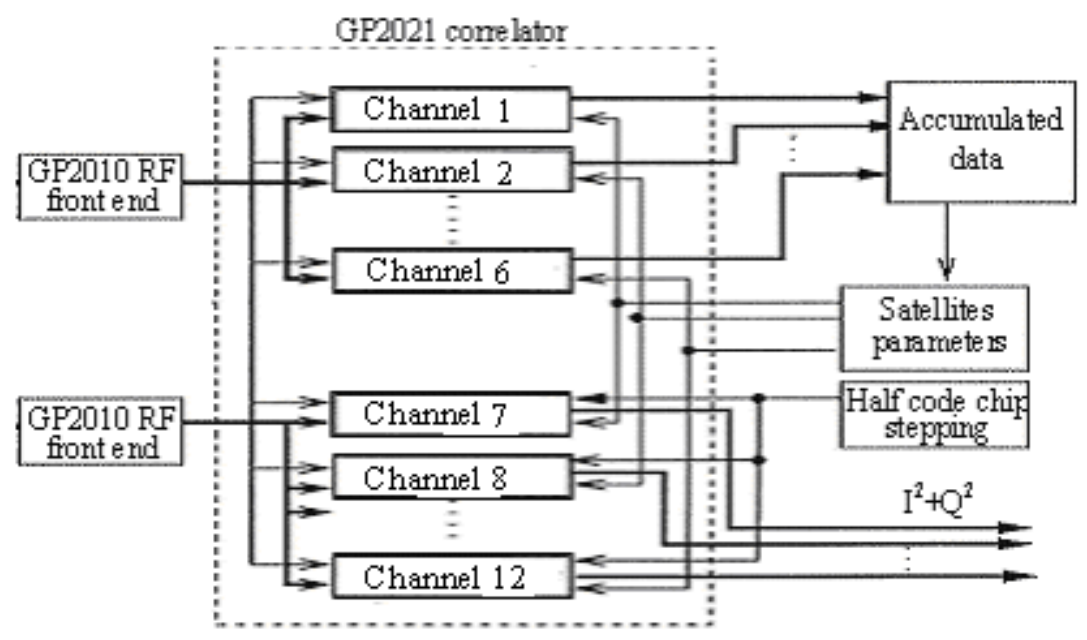

Figure 5. Serial delay mapping receiver (SDMR)

\section{EXPERIMENT RESULTS}

The design and development of SDMR has been finished. The SDMR was equipped in YUN12 and a series of flight tests were done in Tianjin, Dalian and Qingdao harbor of China in August 2004. Experiment and the collected data shows that the SDMR can process the GPS signal successfully and give the correlation power of reflected signal. Figure 6 shows one test result from Tianjin harbor test. For each delay half code chip, one minute from 13:00:00 to 13:01:00 data is given. From the figure we can see that the correlation power of reflected GPS signal are distributed near the specular reflection delay half code chip and diffused to a certain 
extent. The diffused scope is from -2 half code chips to 4 half code chips.

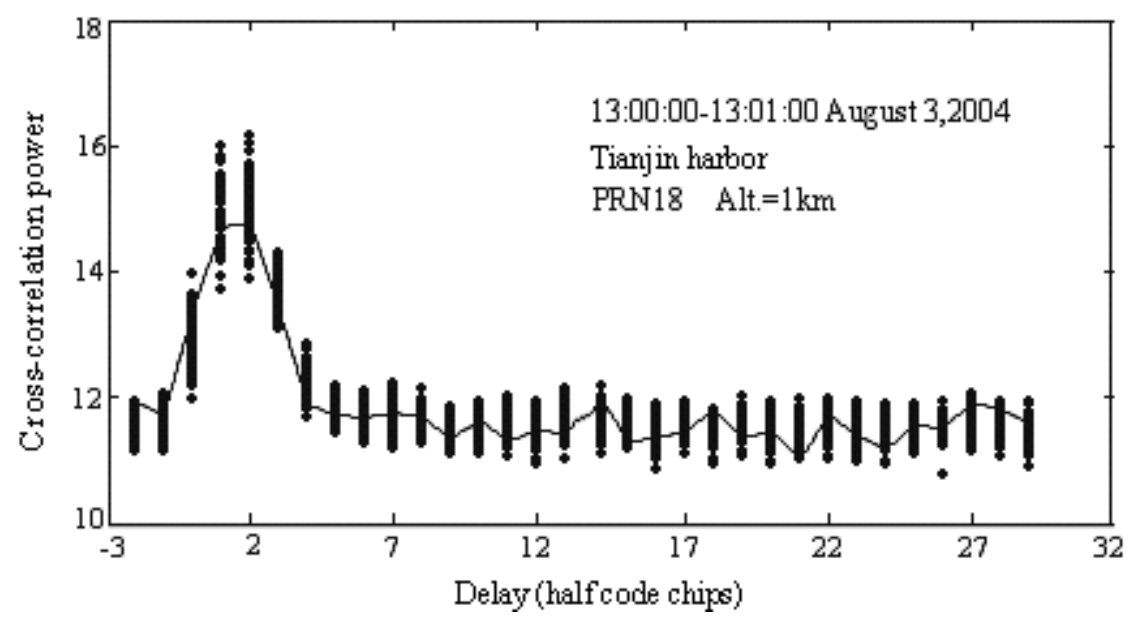

Figure 6. Example of reflected signal correlation power data

\section{CONCLUSION}

DMR based GPS satellite signal is designed and discussed in this paper. This DMR works under serial mode with two antennas and two RF front ends. It provides the user with positioning information and reflected signal cross-correlation power, which can be used to deduce the wind field of sea surface. Test result shows that the design and data processing is correct and valid. Based on the collected data and analysis result the DMR can be also used for detecting sea ice, studying humidity of earth and even the tsunami prediction.

\section{REFERENCE}

[1]Garrison, J. L., S. J. Katzberg and C. T. Howell III: "Detection of Ocean Reflected GPS Signals: Theory and Experiment'. In Proceedings of the IEEE Southeastern '97: Engineering the New Century, pages 290-294, Blacksburg, VA, 1997.

[2]Komjathy, A., V. Zavorotny, P.Axelrad, G. Born, and J. Garrison: "GPS Signal Scattering from Sea Surface between Experimental Data and Theoretical Model". $5^{\text {th }}$ Int. Conf. On Remoting Sensing for Marine \& Coastal Environments, 1: 530-539, 1998.

[3]Zavorotny, V. U. and Voronovich: "Scattering of GPS Signals from the Ocean with Wind Remote Sensing Application”. IEEE Transactions in Geoscience and Remote Sensing, Vol. 38, No. 2, pp. 951-964, 2000.

[4]Elfouhaily, T., B. Chapron, K. Katsaros, and D. Vandermark: "A Unified Directional Spectrum for Long and Short Wind-driven Waves". Journal of Geophysical Research, Vol. 102, pp. 15,781-15,796, 1997. 
[5]Armatys, M., D. Masters, A. Komjathy, P. Axelrad, and J. L. Garrison: "Exploiting GPS as a New Oceanographic Remote Sensing Tool". National Technical Meeting of the U.S. Institute of Navigation, Anaheim, CA, 26-28 January, pp. 339-347, 2000.

[6]Yinfeng Wang: "Development of GPS Receiver Based on DSP Technology". Paper for master of Beihang University, china.1999

[7]Xinyu Luo; "Development of High Dynamic GPS Receiver Based on DSP Technology". Paper for master of Beihang University, china. 2002 